\title{
EDI - ELETRONIC DATA \\ INTERCHANGE - COMO PARCEIRO \\ DE CONFIANÇA NA TROCA DE \\ INFORMAÇÕES ENTRE CLIENTES E \\ FORNECEDORES:
}

\author{
UM Estudo de CASO
}

Ezequiel de Souza Pereira ${ }^{1}$ Flavio Belli

\section{RESUMO}

O objetivo do trabalho em questão foi descrever sobre a comunicação eletrônica de dados (EDI) e apresentar os principais passos para implantação e adequação desse sistema de comunicação dentro de uma indústria metalmecânica localizada em São Bento do Sul, Santa Catarina. O estudo também buscou apontar como a logística das pequenas e médias empresas poderá obter competitividade através do correto uso do sistema EDI. Na coleta e análise dos dados, foi utilizada uma pesquisa de campo, em que foi possível efetuar comparativos entre clientes e fornecedores, que já se utilizavam deste sistema em suas organizações. Quanto aos resultados obtidos neste trabalho, destaca-se a implantação do EDI na unidade TES, fazendo com que o mesmo se tornasse único e exclusivo meio de comunicação junto a todos os clientes, sendo aplicado à grande maioria dos fornecedores. Assim, foi possível elevar o nível de serviço de atendimento mantendo sempre a informação online e confiável.

Palavras-Chave: Comunicação eletrônica. Nível de serviço. Logística. 
$\mathrm{Na}$ constante busca por vantagens competitivas e por estratégias de diferenciação cada vez mais acirradas, as empresas precisam ser mais ágeis e flexíveis no que diz respeito ao atendimento ao cliente. Nas últimas décadas, tem sido grande o esforço para que essa agilidade e flexibilidade no atendimento sejam percebidas de uma forma imediata e diferente. Sobreviver nesse ambiente de mercados econômicos abertos, denominados por Schumpeter (1982) como ciclo da globalização da economia, requer atitudes proaativas por parte das empresas e seus gestores, para não serem descartados.

\section{Nesse contexto, o Intercâmbio Eletrônico}

de Dados (EDI) surge como um tipo de tecnologia de informação capaz de estreitar o relacionamento entre empresas, podendo ser definido como um fluxo eletrônico e padronizado de dados entre empresas, que permite melhorar os resultados, tanto em termos operacionais quanto estratégicos.

Este artigo busca estudar o processo de adoção das novas tecnologias de informação, em que se analisa os impactos provocados pela implantação do EDI nos aspectos organizacionais, naqueles referentes à cultura e aos critérios de desempenho, bem como em relação às atividades comerciais e administrativas.

Em decorrência dessa agressividade competitiva surgem, simultaneamente, necessidades estratégicas de: ter acesso a informações confiáveis; compreender a complexidade dos cenários; programar ações rápidas que permitam o domínio de tecnologias e inovações; viabilizar novos processos mais rentáveis; aplicar modelos de gestão - mais abertos e participativos (SCHUMPETER, 1982); atender às pressões por maior qualificação profissional. Buscar igualar-se no mercado, ou apenas competir por preços, como menciona Goldratt (2007),já não basta. Todos conseguem fazer isso, menciona Drucker (1975), ao mesmo tempo em que se pergunta: qual nosso diferencial, porque os clientes querem comprar nossos produtos?

Quando Morgan (1997) analisa a questão da tomada de decisão nas empresas, no que se refere à adaptação da organização ao ambiente, sugere que o sucesso nessa questão passa por superar as necessidades dos clientes, surpreendê-los, antecipar-se, assunto também amplamente delineado por Kotler (1993).

A partir dessas questões, pode-se questionar como o EDI (Eletronic Data Interchange) auxilia uma organização neste cenário de competitividade?

Para responder a essa questão, conforme cita a Companhia Eletrônica do Paraná (2003) - Celepar, o uso do EDI tem como proposta automatizar as transações comerciais e eliminar o grande número de papéis que são trocados entre empresas.

Ainda segundo a Celepar(2003), esta não deve ser a principal motivação que empurra as empresas no sentido de implantar o EDI em seus negócios e, sim, a busca da competitividade empresarial deve ser a referência. $A$ agilidade e a produtividade são os dois pilares para que uma empresa consiga ser competitiva, nestes tempos de economia globalizada e recessiva.

Este trabalho tem como objetivo geral demonstrar como o EDI e suas partes podem contribuir 
para a melhoria dos resultados operacionais de uma organização no que tange à troca eletrônica de informações como: envio de programações, promessas de entregas e avisos de embarques. Como objetivos específicos, tem-se: descrever os conceitos utilizados na implantação do sistema, elencar os benefícios da comunicação para o fornecedor e para a TES, demonstrar o processo de implantação do sistema EDI e levantar equipamentos necessários para implantação desse sistema.

\section{$2 \mathrm{EDI}$}

O EDI tem sido utilizado como ferramenta estratégica pelas empresas, principalmente na relação cliente-fornecedor, podendo ser definido como o movimento eletrônico de informações entre o comprador e o vendedor, com o propósito de facilitar uma transação de negócios. (HANSEN; HILL, 1989).

O EDI tem permitido a modificação na forma de organização das empresas com respeito aos diferentes processos produtivos, obtendo melhorias na produtividade e na eficiência dos agentes empresariais, podendo também diminuir os custos de produção de uma simples operação, por coordenar atividades de produção entre empresas distintas. Assim, essa tecnologia surge como um diferencial nesses tempos de mudanças, em que a globalização sugere a realização de negócios no mundo inteiro de uma nova maneira, de modo a provocar um equilíbrio positivo entre a qualidade de seus produtos/serviços e as necessidades específicas dos diversos clientes. (DANIELS et al., 1996).

O EDI é uma nomenclatura universal para padronizar Eletronic Data Interchange, conforme a norma ISO 9735. Seus conceitos foram inicialmente desenvolvidos na França, desde 1987, sendo que o número de aplicações vem crescendo rapidamente.
O EDI foi definido pela padronização Francesa (Marcillet, 1994) EDIFRANCE como

$$
\begin{aligned}
& \text { transferência de dados de computador } \\
& \text { para computador, entre parceiros de } \\
& \text { negócios, usando mensagens eletrôni- } \\
& \text { cas de dados, estruturados e agrupa- } \\
& \text { dos, na forma de mensagens padrões, } \\
& \text { desta forma, favorecendo a diminuição } \\
& \text { de custos, e aumentando a produtivi- } \\
& \text { dade da companhia, melhorando os } \\
& \text { procedimentos, e reduzindo custos. } \\
& \text { Esta é uma nova forma de comunica- } \\
& \text { ção entre parceiros econômicos, nos } \\
& \text { quais os documentos em papel não são } \\
& \text { mais necessários. (PRESTON, 1988; } \\
& \text { HINGE, 1988). }
\end{aligned}
$$

As normalizações são de vital importância para que as transações, via EDI, sejam bem sucedidas. Este assunto é um dos principais problemas da implantação do EDI ao longo da Cadeia de Suprimentos, pois as companhias podem adotar padrões diferentes de EDI. (MALO, 1996).

Para resolver os problemas de padronizações, muitas empresas utilizam as VANs (Value Added Network Services). Os dados para uma transmissão através de EDIdevem estar estruturados, tal como: número do produto, nome do cliente, quantidade etc. Devemos diferenciar o EDI do eletronic mail, onde os dados devem ser transmitidos de um ad hoc enquiry, contendo forma não reconhecida. 
O EDI PODE SER VISTO COMO

UM SISTEMA DE COOPERATIVA,

REQUERENDO A COLABORAÇÃO

DE PELO MENOS DUAS PARTES,

USUALMENTE COM DIFEREN-

TES OBJETIVOS DE NEGÓCIOS.

ESTE SISTEMA DE COOPERATIVA

PODE ENVOLVER AS CORPORA-

ÇÕES E SEUS FORNECEDORES

E CLIENTES; CORPORAÇÕES E

SEUS BANCOS, ENTRE AS JOINT

VENTURES DE UMA COMPANHIA,

OU ENTRE COMPETIDORES.

(PRESTON, 1988).

Para muitos, se não para todos os negócios, o EDI é uma etapa inicial radical, que envolve não somente um investimento em hardware $\mathrm{e}$ software, taxas de network e custos de desenvolvimento, entretanto, mais significante ainda, é um re-pensar do relacionamento entre cliente e fornecedor, uma adaptação nas práticas de trabalho, e uma dramática alteração no ciclo de negócios.(PRESTON,1988). Na reposição automática de estoques feita através do EDI, por exemplo, são enviados diariamente para o fornecedor os dados do movimento de saída de seus produtos das lojas dos clientes. $\mathrm{O}$ sistema vai controlando os dados do estoque até que, atingido certo limite predeterminado, ele próprio ordena a reposição desta mercadoria automaticamente, enviando já uma nova remessa de produtos e emitindo as notas fiscais. Dessa forma, elimina-se a necessidade do pedido, podendo ficar a cargo do próprio fornecedor o controle do estoque do cliente. (BITTAR; LIMA, 1997).
O EDI oferece um grande número de vantagens, entre as quais é possível citar:

- economia de tempo, devido ao aumento da velocidade na troca de informações;

- reduções: de pessoal,de papel,de inventário, do tempo para o ciclo de transações comerciais do lead time (WANG; SEIDMANN, 1995);

- qualidade da informação, pois diminui a possibilidade de erros de processamento, durante a troca de informações;

- permite uma ligação muito próxima com o fornecedor;

- confiabilidade e disponibilidade da informação, pois a circulação de informações não depende do movimento físico de documentos, no qual somente o operador que possui o documento tem acesso às informações. (MARCILLET,1994;PRESTON,1988).

\subsection{Históricos do EDI no mundo e no Brasil}

O conceito do EDI não é novo, sua origem vem dos Estados Unidos, quando nos anos 60, vários setores industriais (companhias aéreas, indústria automobilística e saúde) tentaram estabelecer o EDI, mas se comparado com a tecnologia atual, os padrões eram insuficientes e o sistema de comunicação inflexível. No início da implementação do EDI, foram desenvolvidos formatos para atender, isoladamente, às necessidades individuais de cada empresa. Em pouco tempo, os usuários perceberam as limitações desses padrões proprietários e, com isso, novos padrões industriais foram desenvolvidos para atender às necessidades da ampla comunidade de interesse.Alguns dos padrões hoje utilizados, por ordem de número de usuários, são: 
- Padrão CNAB: desenvolvido para operações entre clientes e bancos. Éo mais antigo, tendo sido liberados os primeiros documentos em 1979 pelo Conselho Nacional de Automação Bancária.

- Padrão RND (Rede Nacional de Dados): contemplando os documentos necessários às operações comerciais entre montadoras de veículos, fornecedores de autopeças e bancos. O RND é suportado por um grupo de trabalho misto, Anfavea \& Sindipeças, considerando as necessidades do setor automotivo.Oesforço de padronização do RND foi iniciado no final dos anos 80. Entretanto, sua efetiva utilização vem sendo verificada desde 92, envolvendo hoje a maioria das montadoras brasileiras.

- Padrão NTC:entidade mais representativa das empresas de transporte rodoviário de carga do Brasil.

- Padrão EDIFACT: versão EANCOM. Trata-se de um padrão internacional voltado para os processos de compra e venda de mercadorias e serviços.

O primeiro caso prático de aplicação do EDI deu-se no LACES (London Airport Cargo EDP Scheme), sistema para fretes no aeroporto Heathrow em Londres. Introduzido no início dos anos 70, esse sistema foi bem sucedido e convenceu os descrentes de que segurança e confidencialidade não eram mais itens insuperáveis.

De fato ficou demonstrado que o EDI oferece mais do que um método para manuseio de dados de negócios. Entretanto, tão importante quanto o LACES, muitos fatos foram cruciais para a adoção do EDI. (PRESTON, 1988).

\subsection{EDI no Brasil}

Embora há muito tenha deixado de ser novidade, no Brasil, a prática do EDI encontra-se em estágios distintos, a indústria automobilística e os bancos já utilizam intensamente, outros começam a adotar, pressionados por seus grandes clientes, e muitos ainda mal sabem do que se trata.
A adoção do EDI traz vantagens para os compradores e desvantagens para outros fornecedores. Como resultado, os compra- dores dão um preço prêmio aos fornecedo- res que adotam EDI, e incrementam seus volumes de vendas e compartilhamento de mercado.

Em muitos casos, em que a adoção do EDI é essencial para o comprador conseguir os benefícios desejados, e para os fornecedores, a implantação deste é cara, então, o comprador pode subsidiar o fornecedor nessa implementação.É importante ressaltar que enquanto o EDI reduz os custos de transações dos compradores, tem também como resultado um aumento dos custos diferenciais.

Desconhecimento, curiosidade, receio e necessidade. Esses são os ingredientes básicos de quem opera este serviço pela primeira vez, não necessariamente nesta ordem.

\subsection{EDI na gestão da cadeia de suprimentos (SCM)}

Os ciclos econômicos geram uma troca substancial de documentos em papel entre os vários agentes econômicos, tais como: compradores, fornecedores, transportadores, serviço de abastecimento, administradores, bancos etc. 
No geral, o custo de processamento destes documentos chega a $7,5 \%$ do valor do produto acabado. No gerenciamento da cadeia de suprimentos, onde as informações circulam rapidamente entre longas distâncias, um melhor controle ao longo da cadeia tornase fundamental. Este é o objetivo do EDI. (MARCILLET, 1994).

Figura 1: Diagrama de transmissão do EDI

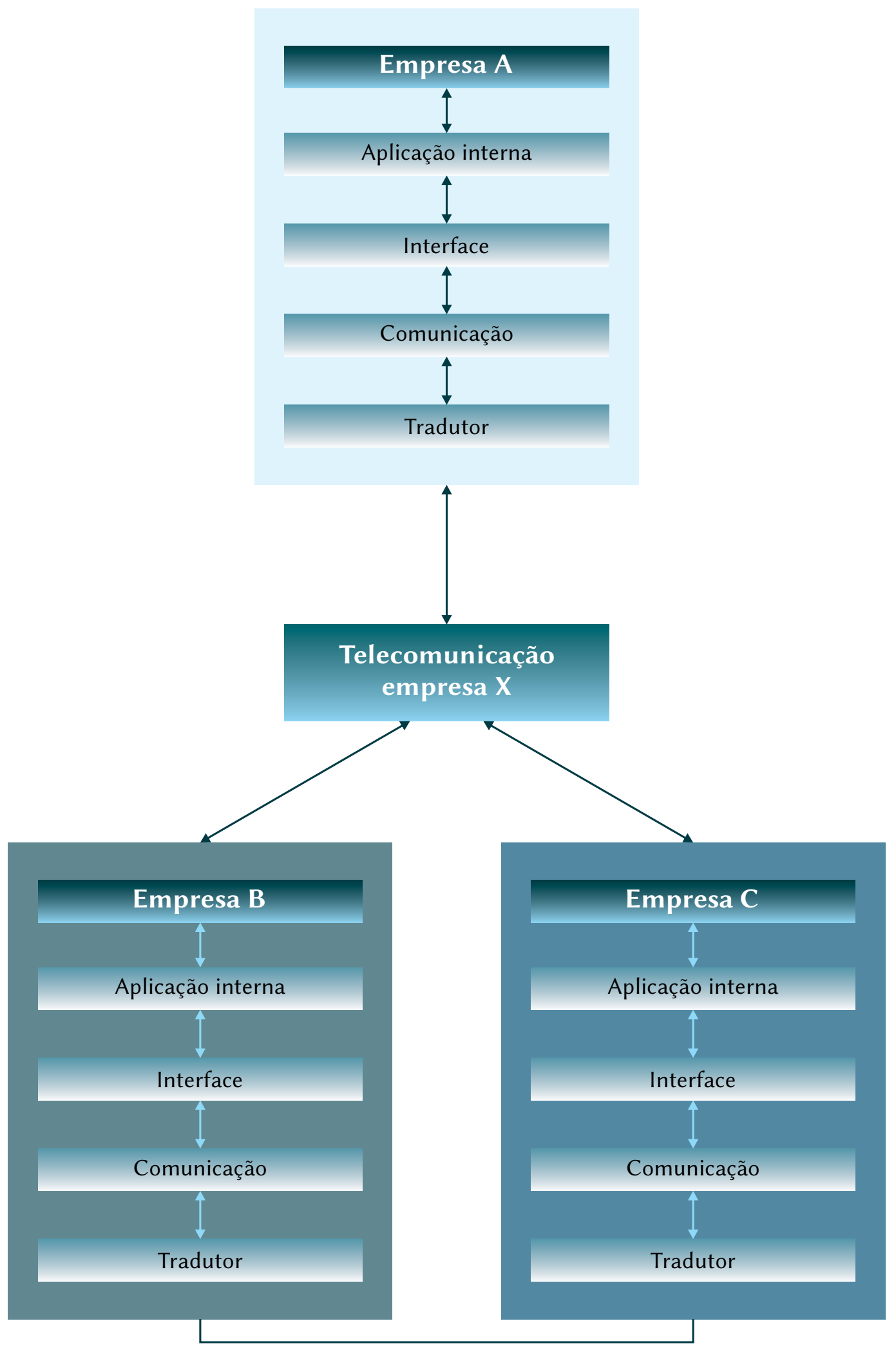

Fonte: Adaptado de Marcillet (1994) 
Muitas companhias implantam o EDI como parte da parceria com seus clientes/fornecedores. Ambos, clientes e fornecedores, esperam ganhar benefícios operacionais e competitivos.

\section{Os BENEFícIOS SURGIDOS COM}

A IMPLANTAÇÃO DO EDI DE-

VEM SER ANALISADOS ENTRE

AS DIVERSAS COMPANHIAS.

Neste CONTEXTO, A figura 1

DEMONSTRA O DIAGRAMA DE

TRANSMISSÃO DE INFORMAÇÕES

VIA EDI ENTRE AS EMPRESAS

QUE FAZEM PARTE DA SUA

CADEIA DE SUPRIMENTOS.

Além da velocidade na transmissão da informação, este meio de comunicação permite a troca de dados estratégicos com maior segurança.

\subsection{Benefícios no uso do EDI}

Os maiores benefícios com o EDI surgem quando sua implantação já está num estágio mais avançado. Neste estágio, clientes e fornecedores trocam informações sobre o modelo da demanda, nível de estoque e planejamento da produção. Alcançar este nível de implementação é um grande desafio para os profissionais envolvidos com a instalação do EDI.

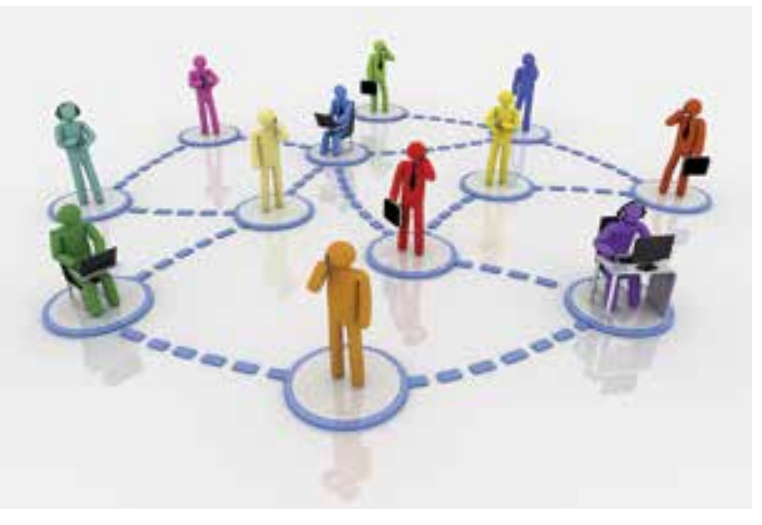

O EDI oferece algumas vantagens, cujos impactos costumam ser maiores do ponto de vista estratégico em detrimento do operacional. Essa tecnologia melhora a eficiência operacional da organização, em razão de possibilitar uma rápida transferência de dados entre empresas e provocar a diminuição dos custos relacionados com papéis, mão de obra e outros custos operacionais.

Além disso, confere vantagem estratégica em virtude de enriquecer a tomada de decisão, por meio de maior previsibilidade e aumento da qualidade das informações processadas com uma menor margem de erros.

\subsection{Delimitação do estudo}

O estudo em questão refere-se à uma empresa do ramo metalmecânico, localizada em São Bento do Sul-SC, que atua como fornecedora de sistemas de exaustão e catalisadores para o mercado de OEM - Original Equipamento Manufacturing.Já está no mercado há treze anos, possui clientes no Brasil e no exterior, tendo como principais clientes: MAN,Mercedes Benz do Brasil, Ford, GM, John Deere, Iveco, entre outras.

Em função do alto grau de exigências dos clientes e da necessidade constante de adequação a essas exigências, sempre se encontram lacunas a serem preenchidas. Frente a isso e tendo como principal objetivo manter-se competitiva, uma comunicação rápida e eficiente é fundamental para a manutenção desses clientes. 


\subsection{Descrição da implantação do EDI na organização}

A empresa em questão desenvolveu a integração entre seus clientes e fornecedores com o objetivo de agilizar a troca de informações comerciais entre empresas, evitando a perda de tempo com a emissão e o manuseio de documentos, aumentando, assim, a confiabilidade e portabilidade das informações. Para a implantação desse sistema foi utilizada a ferramenta PDCA. Segundo De Andrade (2003), o ciclo PDCA foi criado na década de trinta pelo estatístico americano Walter A. Shewhart e é descrito por Moura (1997 apud de Andrade, 2003) como "uma ferramenta que orienta a sequência de atividades para se gerenciar uma tarefa, processo, empresa etc.”, conforme figura 2, Planejar (plan), Executar (Do), Verificar (Check) e Agir (Act).

Figura 2: Quadro PDCA

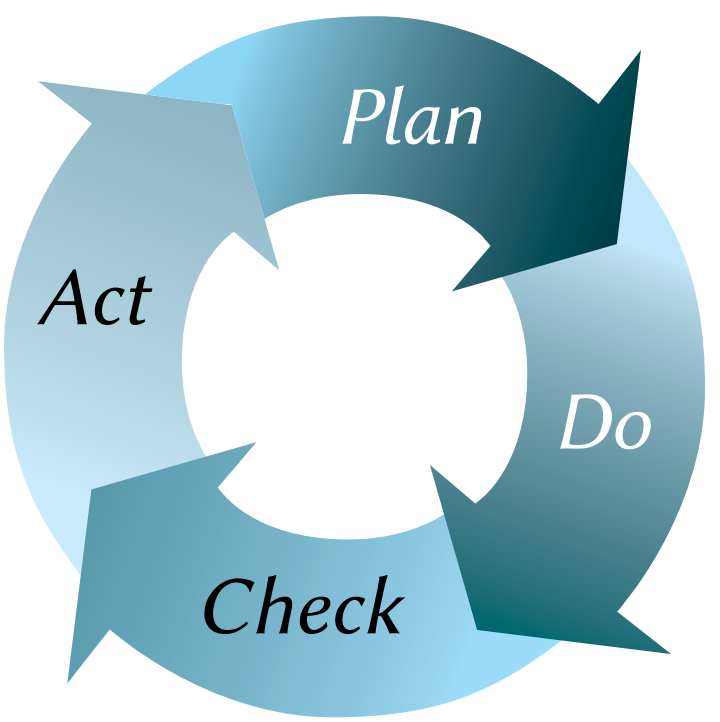

Fonte: De Andrade (2003)

\subsection{Desenvolvimento do}

PDCA para o projeto

implantado

Quadro 1: Desenvolvimento PDCA do projeto

\begin{tabular}{|l|l|}
\hline Planejar & $\begin{array}{l}\text { Difusão entre os clientes e fornece- } \\
\text { dores da importância da implanta- } \\
\text { ção do sistema. } \\
\text { Definição do padrão adotado. } \\
\text { Levantamentodoscustosenvolvidos. } \\
\text { Preparação tecnológica para } \\
\text { atendimento. }\end{array}$ \\
\hline Executar & $\begin{array}{l}\text { Início da implantação técnica da } \\
\text { plataforma. } \\
\text { Ajuste dastabelas conforme padrão } \\
\text { definido (RND, EDIFACT...). } \\
\text { Integração dos módulos a serem } \\
\text { utilizados para automatizar o pro- } \\
\text { cesso (programações, avisos de } \\
\text { embarques, situação de estoque...). }\end{array}$ \\
\hline Verificar \\
\hline Agir & $\begin{array}{l}\text { Análise dos errose incompatibilida- } \\
\text { des geradas na implantação. } \\
\text { Testar cada ponto do processo, } \\
\text { a entrega/recebimento da remessa } \\
\text { junto ao cliente/fornecedor. }\end{array}$ \\
\hline $\begin{array}{l}\text { Disponibilizar recursos exclusivose } \\
\text { verificar as informações trafegadas } \\
\text { via EDI. } \\
\text { A manutenção do EDI ficará sob a } \\
\text { responsabilidade da Logística e TI. }\end{array}$ \\
\hline
\end{tabular}

Fonte: Dos autores (2013)

\subsection{Forma de comunicação}

O meio pelo qual as informações serão trocadas entre as empresas será através da WEB ou STM-400, em que o cliente e o fornecedor poderão viabilizar o processo de qualquer uma das formas. Os padrões utilizados são:

Programação de Entrega: RND Versão 08.

Aviso de Embarque: RND Versão 11. 


\subsection{Informações trafegadas dos clientes para a TES e da TES para os fornecedores}

\subsubsection{Programação de entregas}

Os clientes enviarão para a TES, toda terça-fei$\mathrm{ra}$, as informações de suas necessidades futuras. ATES enviará,toda quarta-feira, pela WEB e/ ou STM400, aos fornecedores, as informações gerais e as datas de remessa e respectivas quantidades, estabelecidas conforme o programa de produção da TES, prevista para o mês e com horizonte conforme disponibilizado por cada cliente. Os fornecedores que escolherem o STM400 também poderão utilizar a WEB para consultas e realizar o upload dos arquivos em formato RND.

\subsection{Informações trafegadas dos fornecedores para a TES e daTES para os clientes}

\subsubsection{Aviso de embarque}

Dos fornecedores para a TES e da TES para os clientes, ocorre o envio de informação dos materiais embarcados, avisos de embarque. Esta informação permite o acompanhamento do material em trânsito, antecipar as divergências a serem encontradas no recebimento e agilizar o processo junto a contas a pagar.

$\mathrm{O}$ envio do arquivo deverá ser efetuado no formato RND pela WEB ou STM40 do Aviso de Embarque. A atualização na WEB poderá ser feita das seguintes maneiras:

- Manual: o fornecedor, após imprimir a nota fiscal e após entrar no site, digitar os dados da nota fiscal no formulário do site.
- Upload: gerar um arquivo no formato RND, e após gerar a nota fiscal, gravar em um diretório, entrar no site e realizar o upload do arquivo.

- STM400: gerar um arquivo no formato RND, e após gerar a nota fiscal, enviar o arquivo para caixa postal da Embratel.

\subsection{Benefícios da}

\section{comunicação para os clientes e fornecedores}

A comunicação via EDI proporciona uma visão mais clara das quantidades recebidas ou em trânsito pela TES (online), além disso, permite visualizar as notas compensadas ou pendentes em contas a pagar (online). Também proporciona a redução do tempo de espera nas filas para desembarque das mercadorias e, como consequência, reduz o custo do transporte. Outro ganho está relacionado à redução das devoluções de mercadorias e à facilidade do processamento da nota fiscal.

\subsection{Benefícios da}

\section{comunicação para TES}

- Interliga-se de maneira direta, ambas as pontas de um processo produtivo;

- agiliza-seoprocessodeenviodeinformações;

- é disponibilizada maior visibilidade das necessidades diárias para os fornecedores;

- otimiza-se a utilização dos recursos de comunicação;

- prioriza o processo produtivo;

- contribui com a avaliação de performance de entregas junto aos clientes;

- melhoria no nível de serviço junto a todos os clientes. 


\subsection{Recursos necessários}

- Micro computador compatível com IBMPC Pentium com mínimo de 512kb memória Ram e 20MB de espaço livre.
- Acesso à Internet.

- Browser I.E 4.0 ou Superior.

\section{CONCLUSÃO}

Neste trabalho foi abordada a troca eletrônica de dados (EDI) a fim de melhorar o que existe em prática hoje no conceito empresarial, fidelizando o cliente, e fazendo com que os parceiros integrem seus sistemas de forma harmoniosa. Ficou clara a demonstração de que a utilização do EDI entre os parceiros de negócios traz muitas vantagens para todos, mas existem muitos problemas a serem sanados para uma implementação completa do EDI, sendo que um dos principais problemas é a falta de uma padronização internacional.

Após levantamento das informações e implantação do sistema junto à empresa, ficou evidente que projetos EDI de sucesso desenvolvem-se em um ambiente de cooperação e entendimento entre os parceiros.

A IMPOSIÇÃO DE UM PADRÃO PROPRIETÁRIO CONSTRANGE O PARCEIRO E DIfICULTA AS NEGOCIAÇÕES NECESSÁRIAS PARA IMPLEMENTAÇÃO EfICAZ DOS PROJETOS.
No início da implantação do projeto, partiu-se com um padrão proprietário, porém a evolução da implantação só foi possível quando utilizamos um padrão de mercado. (WANG; SEIDMANN, 1995). Como resultado, obteve-se um sistema confiável, atendendo às necessidades do mercado atual.

A vantagem do sistema implantado é o baixo nível de infraestrutura necessária, bem como custos de manutenção relativamente baixos.

Um fator limitador do módulo utilizado é o não tratamento de devoluções existentes. Caso venham a ocorrer, estas deverão ser tratadas de forma manual diretamente no ERP da empresa.

Pode-se concluir que esse processo conseguiu alcançar os objetivos propostos, deixando como sugestão futura a implantação também de um módulo específico em que seja possível tratar as confirmações de entregas de mensagens, onde exista confirmação de todo pedido lido pelo fornecedor e de todas as notas fiscais entregues ao cliente.

Acrescentando a esse, deve-se, também, elaborar um módulo em que seja possível tratar as devoluções existentes de forma automática. 
EDI - ELECTRONIC DATA

INTERCHANGE - AS

A RELIABLE PARTNER

DURING EXCHANGE OF

INFORMATION BETWEEN

CUSTOMERS AND

SUPPLIERS: A CASE STUDY

ABSTRACT

The aim of this work was to describe electronic communication data interchange (EDI) and present the main steps for deployment and adaptation of this communication system within the metalworking industry in São Bento do Sul, Santa Catarina. The study also aimed at pointing out how the logistics of small and medium enterprises will gain competitiveness through the proper use of the EDI system. In collecting and analyzing the data was used a Field Survey, where it was possible to make comparisons between suppliers and customers, who have already used this system in theirorganizations. Regarding the results obtained in this work, we highlight the implementation of EDI in the TES unit, causing it to become the sole and exclusive means of communication with all customers, being applied to the majority of suppliers. Thus, it was possible to raise the level of customer service while maintaining online information reliable.

KEYWORDS: Electronic

Communication. Service Level.

Logistics.

\section{REFERÊNCIAS}

BITTAR, RCSM; LIMA, Paulo Corrêa. A utilização do EDI (Eletronic Data Interchange) como uma ferramenta de integração entre os parceiros de negócios. Anais do XVII Enegep (Encontro Nacional de Engenharia de Produção), 1997.

CELEPAR - Companhia de Tecnologia da Informação e Comunicação do Paraná, 2003. Disponível em: <http://www.celepar.pr.gov.br>. Acesso em: 19 jan. 2013.

DANIELS, J. L.; DANIELS, N. C. Visão global: criando novos modelos para as empresas do futuro. São Paulo: Makron Books, 1996.

DE ANDRADE, Fábio Felippe; MELHADO, Silvio Burrattino. O método de melhorias PDCA. 2003. Tese de Doutorado. Escola Politécnica da Universidade de São Paulo.

DRUCKER, Peter F. Administração: tarefas, responsabilidades, práticas. São Paulo: Pioneira, 1975.

GOLDRATT, Eliyahu M. A Meta: um processo de melhoria contínua. tradução CORBETT NETO, Thomas. 2. ed. São Paulo, Nobel, 2007.

HANSEN, James V.; HILL, Ned C. Control and audit of electronic data interchange. MIS Quarterly, p. 403-413, 1989.

HINGE, Kathleen Conlon. Electronic data interchange: from understanding to implementation. AMA Membership Publications Division, American Management Association, 1988.

KOTLER, Philip. Administração de Marketing: Análise, Planejamento, Implementação e Controle / 2. ed. São Paulo: Ed. Atlas, 1993.

MALO, N. R. EDIFACT or EDIFiction?. Actionline, March 1996, pp. 32-33.

MARCILLET, J. Customer Information Systems - Eletronic Data Interchange. Rail International, Agosto, 1994, p. 24-39. 


\section{SOBRE OS AUTORES}

MORGAN, Gareth. Imagens da organização. São Paulo: Atlas, 1996.

PRESTON, M. What is EDI? The National Centre for Information Technology, Manchester, England, 1988.

SCHUMPETER, Joseph Alois. Teoria do desenvolvimento econômico. São Paulo: Abril Cultural, 1982.

WANG, E. T. G.; SEIDMANN, A. Electronic Data Interchange: Competitive Externalities and Strategic Implementation Policies. Management Science / v. 41, n. 3, march 1995.

Data de recebimento: 20/08/13

Data de aprovação: 20/11/14

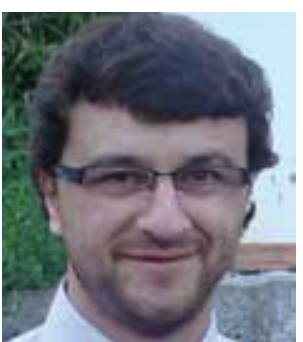

Ezequiel de Souza

Pereira

Tecnólogo em Gestão da Produção e Logística na Univille - Universidade da Região de Joinville. Possui MBA em Logística Empresarial na FGV Fundação Getúlio Vargas e é pós graduado em Gestão da Produção e Logística pela Univille. Possui experiência como profissional de logística no grupo TUPER S.A.

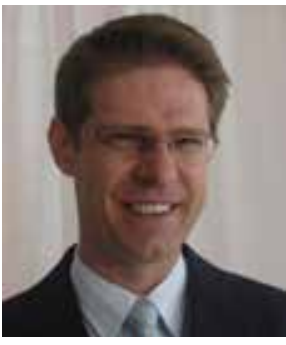

\section{Flavio Belli}

Especialista em Logística Empresarial pelaUnifae egraduado em Administração de EmpresaspelaUniversidadeda Região de Joinville (Univille). Possui experiência como docente universitário das disciplinas de Logística de Suprimentos,Logística de Transporte, Gestão deProjetos Industriais, Gestãoda Produção, Gestão de Pessoas, entre outras. Atua na área de planejamento daTigre S.A.Tubos e Conexões, onde desenvolve o projeto do Lean Management. 